\title{
Identificación de los primeros estados de phyllosoma de la langosta de Juan Fernández (Jasus frontalis) mantenidos en laboratorio*
}

\author{
Enrique Dupré M. y Chita Guisado A. \\ Departamento de Biología Marina, Facultad de Ciencias del Mar \\ Universidad Católica del Norte, Casilla 117, Coquimbo, Chile.
}

\begin{abstract}
RESUMEN. El primer estado larval después de la eclosión corresponde a un naupliosoma que presenta un cuerpo ovoídeo conformado por un cefalón curvado desde el cual se proyectan dos cápsulas oculares curvadas, antenas y anténulas, un pereión curvado y pereiópodos que están enrollados sobre sí mismos. Sólo las anténulas se han extendido y están provistas de setas plumuladas, las cuales le permiten la natación hasta que se produce la muda para originar la primera phyllosoma.

El estado I de phyllosoma presenta un cuerpo transparente y aplanado dorso-ventralmente, ojos no pedunculados, seis pares de setas plumuladas en el exopodito del primer y segundo pereiópodo; exopodito del tercer pereiópodo rudimentario; pereiópodos cuarto y quinto ausentes. En el estado II aparece el pedúnculo ocular, el primer y segundo par de pereiópodos con 7 pares de setas en el exopodito, cuarto par rudimentario. El estado III presenta 8 pares de setas en los exopoditos del primer y segundo pereiópodos y 3 pares en el tercero; cuarto par con longitud de 1 a 1,5 veces el largo del abdomen; quinto par ausente. El estado IV presenta dos sub-estados: A y B. El IV-A tiene un cuarto pereiópodo cuya longitud es 2 veces el largo del abdomen y un tercer pereiópodo con un exopodito provisto de 4 ó 5 pares de setas. En el estado IV-B la longitud del cuarto pereiópodo es 3,5 a 4 veces mayor que el abdomen y el exopodito del tercer pereiópodo presenta 5 pares de setas. En el estado V los dos primeros pares de pereiópodos presentan 10 pares de setas. El exopodito del tercer par de pereiópodos presenta 6 pares de setas. El quinto par es rudimentario.

La sobrevivencia de cada uno de los estados es variable y va aumentando a medida que avanza el desarrollo. La menor sobrevivencia ocurrió en el estado I (17\%) y la mayor en el estado V (90,5\%). La duración total de los cinco primeros estados varió entre los 40 y 57 días y la duración promedio de cada uno de éstos, en un rango de temperatura de $18-23^{\circ} \mathrm{C}$, fueron $13,11,6,19,5$ y 8 días desde el estado I al V respectivamente. La máxima duración de un período de intermuda fue de 27 días. Estos resultados permiten sugerir que el desarrollo larval de Jasus frontalis tendría una duración entre 112 y 160 días y un tiempo máximo de 378 días a una temperatura entre 18 y $23^{\circ} \mathrm{C}$.
\end{abstract}

Palabras claves: phyllosomas, diagnosis, primeros estados, duración estados, intermuda.

\section{Early stages of phyllosoma of the spiny lobster of Juan Fernandez (Jasus frontalis) mantained in laboratory conditions*}

\footnotetext{
ABSTRACT. The first stage after the larval eclosion corresponds to a naupliosome. It presents an ovoid body conformed by curved eyes, pereion and pleon and pereiopods that are wound on themselves. The antennula is the only extended appendage. It is provided with plumose setae which permit the swimming of the naupliosome until the moult takes place originating the first phyllosoma.

The stage I of phyllosoma presents a transparent and flattened dorso-ventrally body. The eyes are non stalked. The stages I and II of phyllosoma have exopods with 6 pairs of plumose setae. The exopod of the third pereiopod is rudimentary and the fourth and fifth pairs of pereiopods are absent. In the stage II of phyllosoma the eye stalk appears:
}

\footnotetext{
* Estudio financiado por el proyecto FONDECYT Nº194-1121.
} 
Investigaciones Marinas

the first and second pairs of pereiopods bear 7 pairs of plumose setae on the exopodito, the fourth pair is rudimentary and the fifth is absent. The stage III of phyllosoma presents 8 pairs of plumose setae on the exopods of the first and second pereiopods and 3 setae in the third pair. The fourth pair is 1 to 1.5 times longer than the abdomen. Fifth pair is absent. The stage IV of phyllosoma presents two sub-stages: A and B. The sub-stage IV A has a fourth pereiopod 2 times longer than the abdomen and a third pereiopod with an exopod with 4 or 5 pairs of plumose setae. The sub-stage IV B has a fourth pereiopod 3.5 to 4 times longer than the abdomen and the exopod of the third pereiopod presents 5 pairs of plumose setae. The stage $\mathrm{V}$ of phyllosoma, the exopods of the first and second pairs of pereiopods present 10 pairs of setae. The exopod of the third pair of pereiopods presents 6 pairs of plumose setae. The fifth pair is rudimentary.

The survival of larvae in each stage is variable and increases as the development advances. The minor survival occurred in the first stage of phyllosoma (17\%) and the mayor survival $(90,5 \%)$ was found in the stage $\mathrm{V}$ of phyllosoma. The total duration of the first 5 stages varied between 40 to 57 days and the duration average of each stage, in a range of temperature of $18-23^{\circ} \mathrm{C}$, were $13,11,6,19.5$ and 8 days respectively. The maximum duration of intermoul period of the larvae was 27 days. These results suggest that the larval development would have a duration between 112 to 160 days and a maximum time of 378 days at temperature between 18 and $23^{\circ} \mathrm{C}$.

Key words: Phyllosoma, early stages, intermoult, stage duration.

\section{INTRODUCCION}

El desarrollo larval parece ser una etapa crucial en el cultivo de los crustáceos. Durante esta etapa ocurre la mayor mortalidad. Los factores que la causan están asociados principalmente con la alimentación y la temperatura, las que influyen sobre la frecuencia de mudas y el crecimiento larval. Sin embargo, existen otros factores que son igualmente importantes de considerar como es la calidad del vitelo de los ovocitos, ya que de éste dependerá el buen desarrollo de los embriones y una mejor sobrevivencia de larvas recién eclosionadas.

El conocimiento sobre la biología del desarrollo de la langosta de Juan Fernández, Jasus frontalis, aunque ha aumentado en los últimos años, aun es escaso. Se ha establecido la duración de cada uno de los estados del desarrollo embrionario a diferentes temperaturas (Dupré, 1988a), lo cual ha permitido predecir el momento de apareamiento de las hembras y el período en que eclosionarán sus larvas por medio de la determinación del estado de desarrollo de los embriones portados por la hembra y conociendo la temperatura del agua a la que han sido incubados. Sin embargo, las larvas obtenidas en muestreos planctónicos no se podían identificar y menos aún conocer su edad y estado de desarrollo, aun cuando existen estudios anteriores sobre los primeros estados de su desarrollo larval (Dupré, 1988b).

Las únicas especies de la familia Palinuridae para las que se ha descrito el desarrollo larvario completo son: Panulirus interruptus con 11 estados de phyllosoma, basado en material planctónico preservado (Johnson, 1956), P. japonicus por Kittaka y Kimura (1989), Palinurus elephas por Kittaka e
Ikegami (1988) y Jasus lalandii con 11 estados (Silberbauer, 1971). En la mayoría de los estudios sobre larvas del género Jasus se han descrito sólo algunos estados del desarrollo larval (Johnson, 1971; Dexter, 1972; Báez, 1973; McWilliam y Phillips, 1987). En Jasus frontalis sólo se conocen los estados VIII, XI y XIII (etapas A y B) descritos por Báez (1973) de larvas capturadas en el plancton. Recientemente se describió bajo microscopía electrónica de barrido el primer estado de phyllosoma obtenido de cultivos en estanques (Dupré, 1996). En base a estos antecedentes se puede inferir algunas características de los estados intermedios. Sin embargo, es indispensable establecer con exactitud las características de cada uno de los estados. Es por esto que el presente estudio describe las características generales de los cinco primeros estados del desarrollo larval y la duración de cada uno de ellos.

\section{MATERIALES Y METODOS}

Varias hembras ovígeras de Jasus frontalis capturadas en el archipiélago de Juan Fernández (33³8'S, $78^{\circ} 50^{\prime} \mathrm{W}$ ) a $60 \mathrm{~m}$ de profundidad con embriones en los dos últimos estados del desarrollo (Dupré, 1988a) fueron transportadas por vía aérea hasta el laboratorio de cultivos de la Universidad Católica del Norte, Sede Coquimbo. Estas se mantuvieron en estanques circulares de 1.000 litros con flujo de agua de mar filtrada a $50 \mathrm{~m}$ y burbujeo constante de aire hasta la eclosión de las larvas. La recolección de larvas se realizó a través de un tamiz de 100 m ubicado a la salida del estanque que contenía a las hembras ovígeras. Grupos de 10 larvas fueron transferidas a recipientes redondos de plástico transparente 
que contenían agua de mar filtrada. El recambio de agua y la alimentación con nauplius de Artemia en exceso se realizó diariamente. Las larvas fueron mantenidas entre diciembre de 1994 y marzo de 1995 a temperatura ambiente la cual varió entre 18 y $23^{\circ} \mathrm{C}$.

La medición de la velocidad de natación se realizó introduciendo las larvas en un tubo de vidrio de 1,2 $\mathrm{m}$ de longitud y $6 \mathrm{~cm}$ de diámetro. Para medir la velocidad se ubicó una fuente de luz en uno de los extremos y se determinó la distancia recorrida por las larvas en una unidad de tiempo.

Las fotografías se realizaron en un microscopio Nikon SMZ-10 y un equipo automático de fotografía Nikon Microflex FX-35. En las descripciones se utilizó la terminología empleada por McWilliams y Phillips (1987) para larvas phyllosoma del género Jasus.

\section{RESULTADOS}

\section{Naupliosoma}

La larva recientemente eclosionada corresponde a un estado de naupliosoma (Fig. 1). Estas son liberadas por medio del movimiento sincrónico y rápido de los pleópodos de la hembra después de extender el abdomen y levantar el telson hacia la superficie lo cual ocurre durante las primeras horas de la noche. El aspecto general del naupliosoma es ovalado. El escudo cefálico, pereión y abdomen están curvados y los apéndices enrollados debajo del escudo cefálico. Los globos oculares están curvados y levemente separados del cefalón (Fig. 1a). Las anténulas están provistas de setas plumuladas. El exopodito presenta seis setas y el endopodio 2 de éstas (Fig. 1b). Después de ser liberadas, las larvas son incapaces de tener movimiento propio por lo cual son arrastradas por corrientes o caen al fondo del estanque, ya que el batir de las anténulas es muy débil. Sin embargo, entre 3 a 15 min post-eclosión el batir de las anténulas se va incrementando hasta lograr una activa natación. En este estado presentan un fuerte fototactismo positivo. Ninguno de los pereiópodos presenta exopodito. Después de $20 \mathrm{~min}$ de natación el naupliosoma se desprende de la primera muda por medio de movimientos de los pereiópodos y origina el primer estado de phyllosoma.

\section{Aspectos generales de los primeros estados de phyllosoma}

Los tres primeros estados de phyllosoma presentan fototactismo positivo al igual que el naupliosoma. La phyllosoma I es fuertemente fototrópica, lo cual va disminuyendo desde éste al III. En el transcurso de los dos primeros estados, la mayor parte del tiempo las larvas permanecen nadando activamente tocando la superficie del agua con sus exopoditos nadadores. En cambio, en los estados III al V nadan activamente entre la superficie y el fondo. La velocidad de natación de la phyllosoma I varía entre 0,54 $\mathrm{m} / \mathrm{min}$ y $0,75 \mathrm{~m} / \mathrm{min}$, pudiendo aumentar a $0,87 \mathrm{~m} /$ min en lapsos de 8 a $12 \mathrm{seg}$.

La morfología externa del escudo cefálico, longitud del pedúnculo ocular, el número de pares de pereiópodos y especialmente el número de setas de los exopoditos natatorios de los pereiópodos, varían desde el estado I al V (Tabla 1).

El tiempo transcurrido entre el primer y quinto estado de desarrollo larval fue de 40 días a una temperatura de $18-23^{\circ} \mathrm{C}$ (Tabla 2 ).

\section{Phyllosoma I}

El primer estado (Fig. 2a) difiere notoriamente del naupliosoma. La longitud total varía entre 2,0 y 2,2 $\mathrm{mm}$ y la longitud del cefalón es de 1,6 mm. Presenta un cuerpo transparente y aplanado dorsoventralmente. En visión lateral las piezas bucales sobresalen notoriamente en la región antero-ventral (Fig. 2b). Presenta sólo 3 pares de pereiópodos. Los dos primeros tienen un exopodito con 6 pares de setas plumosas; en cambio el exopodito del tercer par es rudimentario. Los ojos son elongados pero no pedunculados. Antenas unirrameadas. Anténulas bífidas o birrameadas. El abdomen que es rudimentario y termina en dos prolongaciones laterales. Cada una de éstas termina en una espina cónica con tres setas a su alrededor. Los maxilípedos I y II tienen 4 segmentos y no presentan exopodito.

\section{Phyllosoma II}

Pedúnculo ocular presente (Fig. 3a). Su longitud es 0,3 a 0,4 veces el largo del ojo. El primer y segundo par de pereiópodos con exopodito provisto de 7 pares de setas. El tercer par de pereiópodos presenta 2-3 cromatóforos puntiformes sub-terminales en su extremo distal y un exopodito rudimentario, cuya longitud no es mayor que la del abdomen, pero sí de mayor longitud que en el estado anterior. El cuarto 


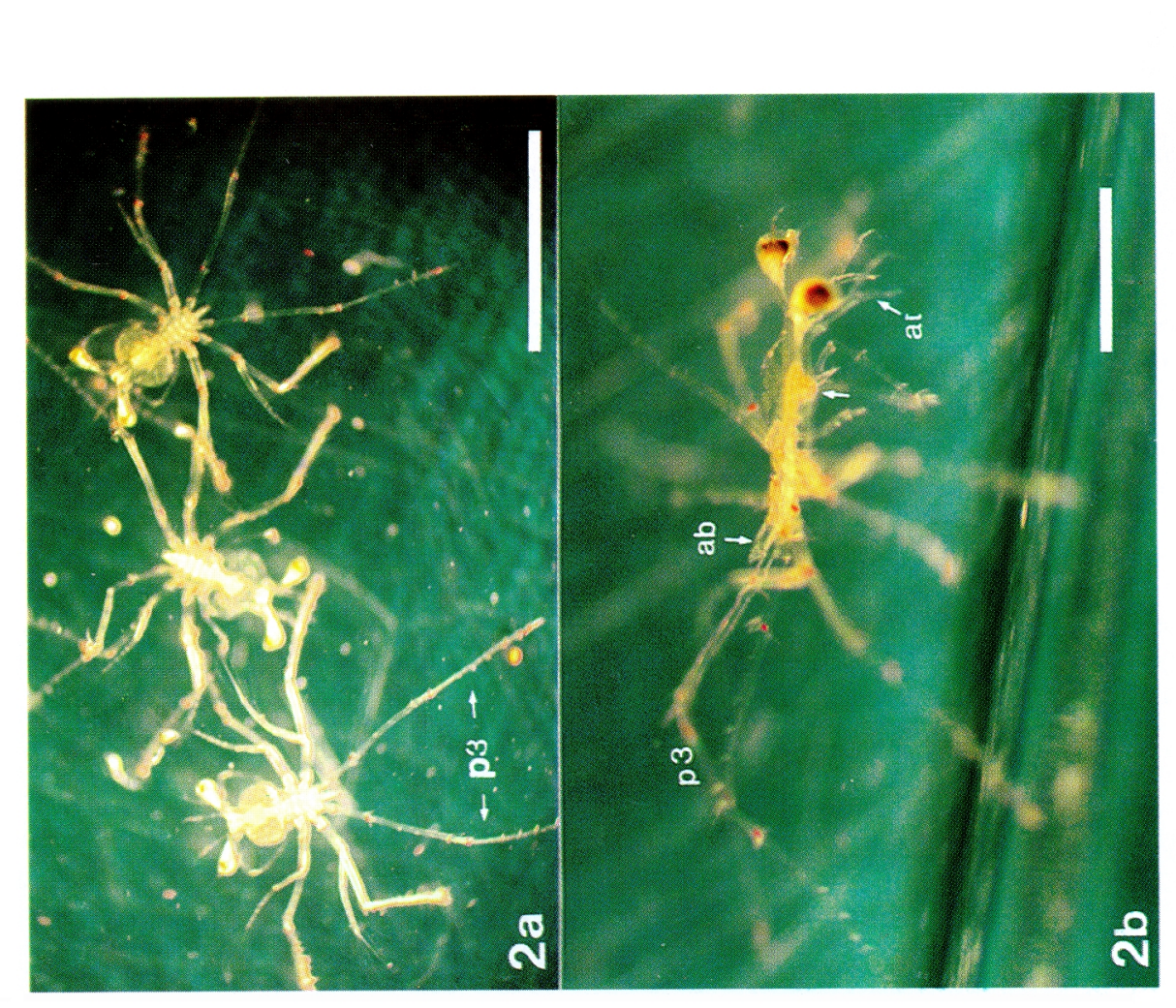

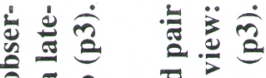

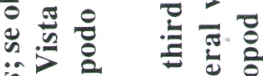
苞定高

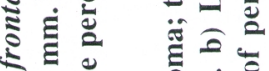
क

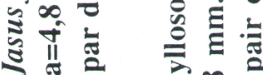
क

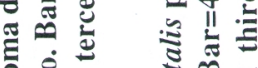

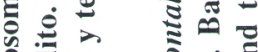

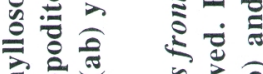

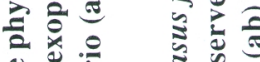
สำ

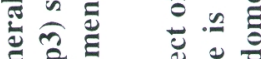

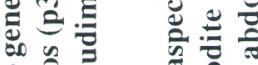
के

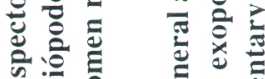

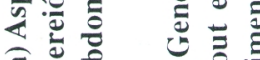
สิ ๘

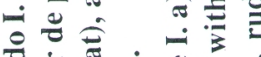

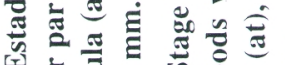

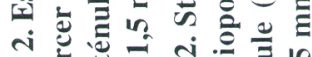

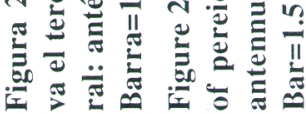
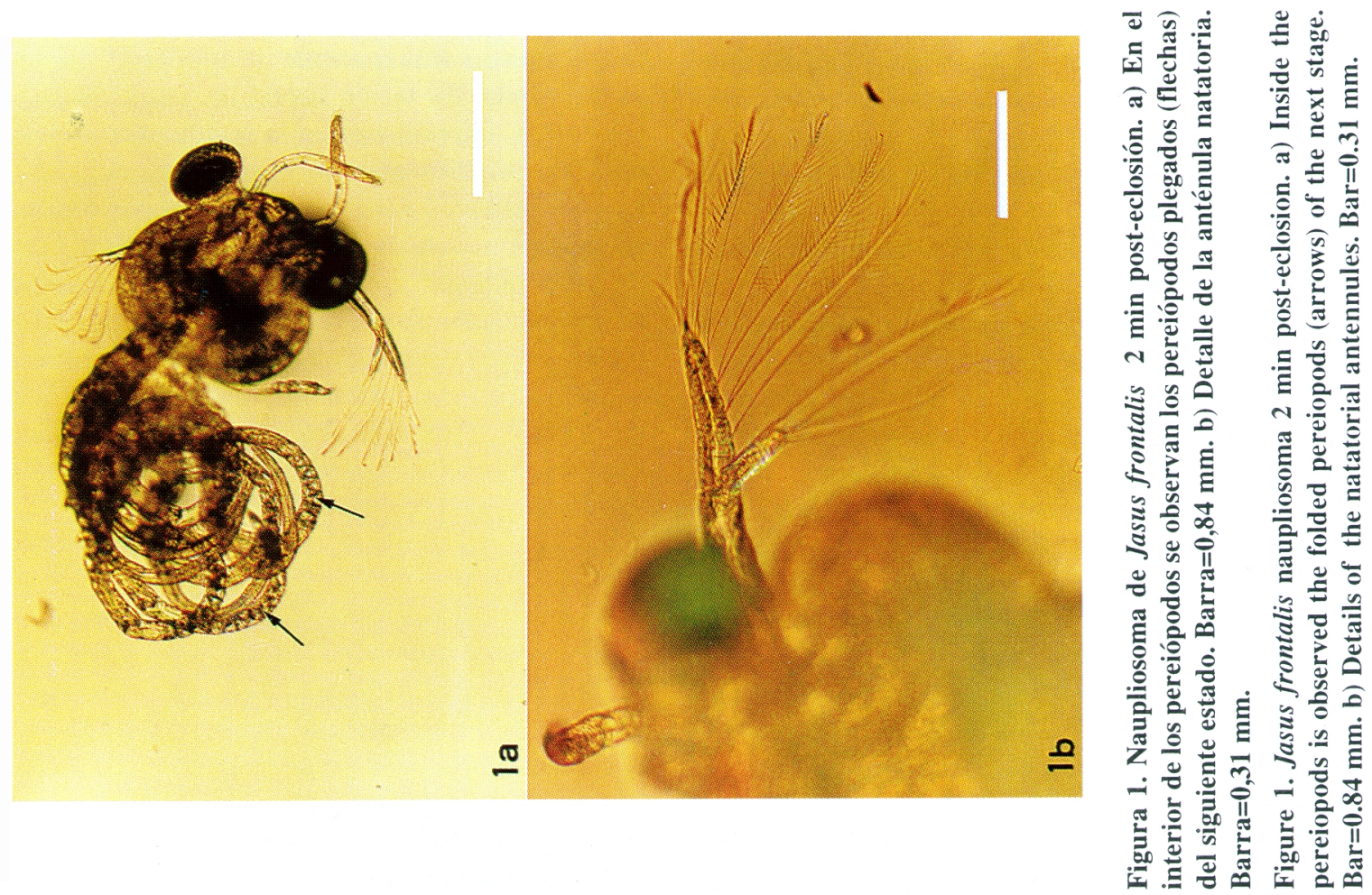

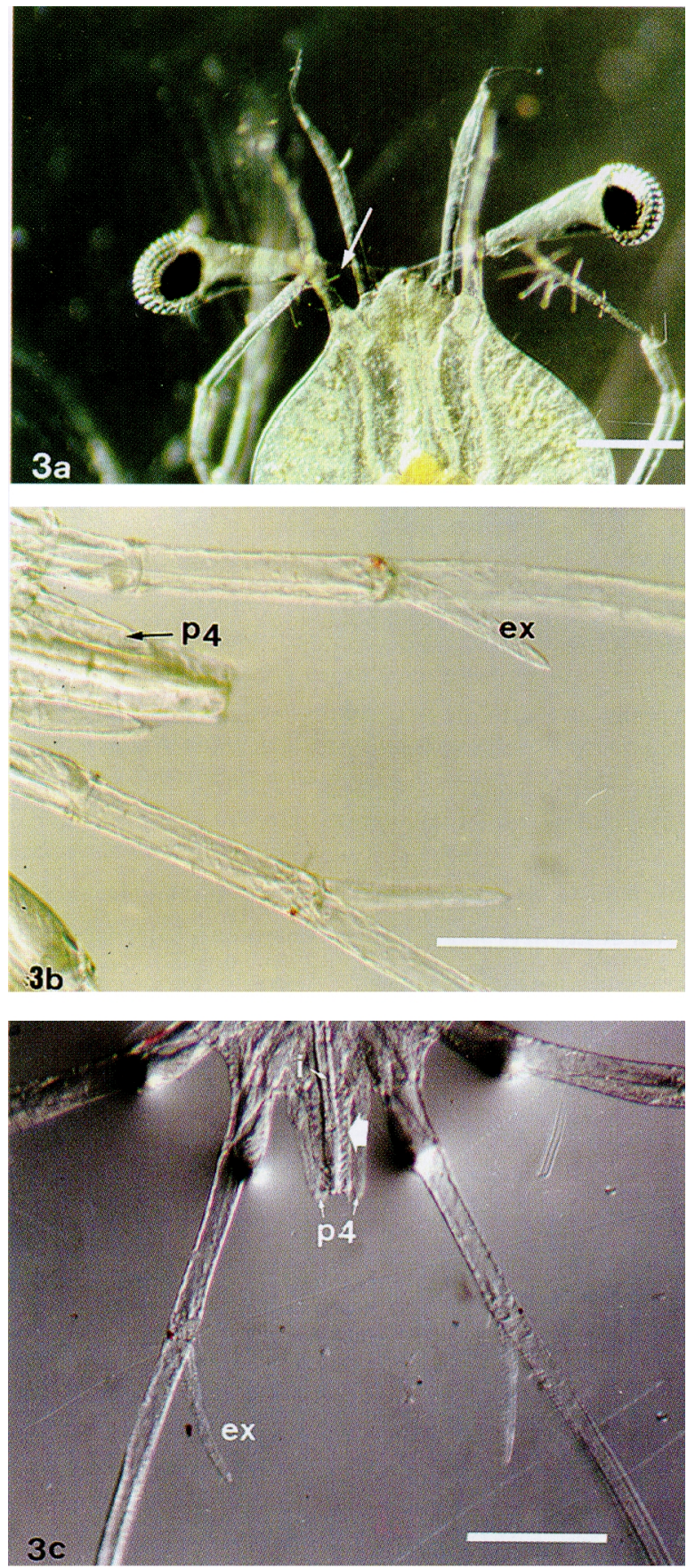

Figura 3. Estado II de phyllosoma. a) Extremo anterior de la larva; se observa pedúnculos oculares (flecha), cápsulas oculares, antena y anténula. b) y c) Extremo posterior de la larva; se observa tercer pereiópodo con su exopodito (ex) rudimentario y el cuarto par de pereiópodos (p4) a ambos lados del abdomen; note la diferencia en longitud del exopodito del tercer y cuarto pereiópodo. Barras=0,5 mm.

Figure 3. Stage II of phylosoma. a) Larvae in anterior view; ocular peduncles are observed (arrows), ocular capsules, antenna and antennule. b) and c) Larvae in posterior view; it's observed the third pereiopod with its rudimentary exopodite (ex) and the fourth pair for pereiopods (p4) in both sides of the abdomen; note the difference of lenght in the exopodite of the third and fourth pereiopod pair. Bars $=0.5 \mathbf{~ m m}$. 
Tabla 1. Características de los 5 primeros estados (P1 a P5) de phyllosoma de la langosta Jasus frontalis. Table 1. Characteristics of Jasus frontalis lobster first 5 phyllosoma stages.

\begin{tabular}{|c|c|c|c|c|c|c|c|c|c|c|c|}
\hline \multirow[t]{2}{*}{ Estados } & \multicolumn{2}{|c|}{ P1 } & \multicolumn{2}{|c|}{$\mathbf{P 2}$} & \multicolumn{2}{|c|}{ P3 } & \multicolumn{2}{|c|}{ P4 } & \multicolumn{2}{|c|}{ P5 } & \multirow[t]{2}{*}{ Poc } \\
\hline & En & Ex & En & Ex & En & Ex & En & Ex & En & Ex & \\
\hline I & $\mathrm{P}$ & $6-7$ & $\mathrm{P}$ & $6-7$ & $\mathrm{P}$ & Rud & - & - & - & - & - \\
\hline II & $\mathrm{P}$ & 7 & $P$ & 7 & $\mathrm{P}$ & Rud & Rud & - & - & - & $\mathrm{P}$ \\
\hline III & $\mathrm{P}$ & 8 & $P$ & 8 & $\mathrm{P}$ & 3 & $2 s$ & - & Rud & - & $\mathrm{P}$ \\
\hline IV A & $\mathrm{P}$ & 9 & $P$ & 9 & $\mathrm{P}$ & 4 & $3 \mathrm{~s}$ & - & Rud & - & $\mathrm{P}$ \\
\hline IV B & $\mathrm{P}$ & 9 & $\mathrm{P}$ & 10 & $\mathrm{P}$ & 5 & $3 \mathrm{~s}$ & - & Rud & - & $P$ \\
\hline V & $\mathrm{P}$ & 10 & $P$ & 10 & $\mathrm{P}$ & 7 & $4 s$ & Rud & $\mathrm{P}$ & 1 & $\mathrm{P}$ \\
\hline
\end{tabular}

En = endopodito presente; $\mathrm{Ex}=$ pares de setas sobre el exopodito; $\mathrm{P} 1$ a P5 = pereiópodos del primero al quinto; $\mathrm{P}=$ presente desde este estado; Poc. = pedúnculo ocular; $\mathrm{Rud}=$ pudimento; $-\stackrel{=}{=}$ ausencia de la estructura; $\mathrm{s}=$ segmentos.

Tabla 2. Duración y sobrevivencia de los primeros estados del desarrollo larval de la langosta Jasus frontalis.

Table 2. Duration and survival of the first stages of Jasus frontalis lobster larval development.

\begin{tabular}{|c|c|c|c|c|}
\hline \multirow{2}{*}{$\begin{array}{l}\text { Estado de } \\
\text { phyllosoma }\end{array}$} & \multicolumn{2}{|c|}{ Duración (días) } & \multirow{2}{*}{$\begin{array}{l}\text { Sobrevivencia } \\
\text { por estado }(\%)\end{array}$} & \multirow{2}{*}{$\begin{array}{c}\text { Tiempo } \\
\text { acumulado }\end{array}$} \\
\hline & Promedio & Rango & & \\
\hline I & 13 & $12-14$ & 17,0 & $12-14$ \\
\hline II & 11 & $9-13$ & 34,0 & $21-27$ \\
\hline III & 6 & $5-7$ & 50,0 & $26-34$ \\
\hline IV & 10,5 & $9-12$ & 90,7 & $35-46$ \\
\hline $\mathrm{V}$ & 8 & $5-11$ & 92,5 & $40-57$ \\
\hline
\end{tabular}

par de pereiópodos es rudimentario y se observan a ambos lados del abdomen. Su extremo distal no sobrepasa el tercio distal del abdomen (Fig. 3b). Antenas, maxila II y maxilípedo I no presentan variación. Algunas larvas al pasar a este estado presentan variaciones en cuanto a la longitud del exopodito del tercer y cuarto par. El exopodito del tercer par, así como el cuarto par de pereiópodos tienen una longitud mayor que el abdomen (Fig. 3c). El extremo distal de todos los pereiópodos presenta pigmentación rojo-anaranjada, detrás de la cual existe un cromatóforo puntiforme de color obscuro. Base de la antena con un cromatóforo rojo ramificado. Borde anterior del cefalón con una mancha naupliana.
Anténula con 3 espinas en su extremo distal: una central fuerte y 2 laterales muy pequeñas. Primera maxila fuertemente pigmentada.

\section{Phyllosoma III}

Primer y segundo par de pereiópodos con 8 pares de setas. El tercero presenta 3 segmentos y su exopodito posee 3 pares de setas plumuladas (Fig. 4). El cuarto par presenta 2 segmentos y tiene una longitud equivalente a 1-1,5 veces la longitud del abdomen (46,5 a $70 \mu \mathrm{m})$; no presenta exopodito y termina en 2 espinas divergentes. El rostro se extiende hacia adelante sin sobrepasar la inserción de los pedúnculos oculares. La longitud de los 


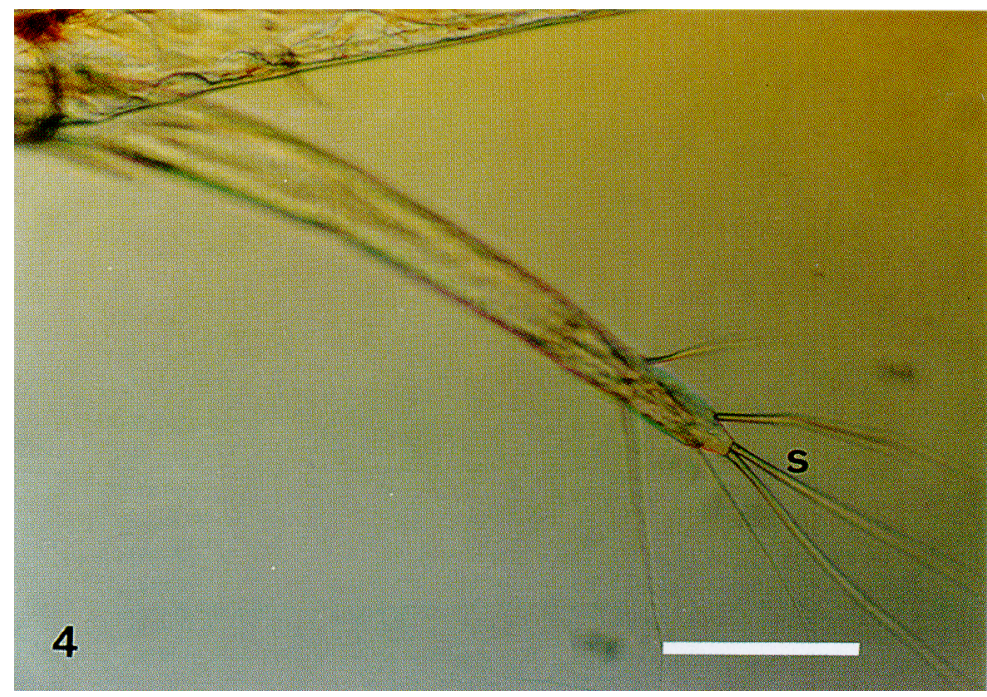

Figura 4. Estado III. Extremo distal del exopodito del tercer pereiópodo con 3 pares de setas $(\mathrm{s})$. Barra $=0,15 \mathrm{~mm}$.

Figure 4. Stage III. Distal end of the exopodite in third pereiopod with 3 pairs of setaes (s). Bar $=0.15 \mathrm{~mm}$.

pedúnculos oculares es 0,7 a 0,9 veces el largo del ojo (Fig. 5a). La antena presenta un esbozo de endopodito con 2 setas terminales (Fig. 5b). La anténula termina en 4 espinas largas y 2 más cortas en posición dorsal; su borde interno está provisto de una o dos setas largas y una espina dentada más atrás.

\section{Phyllosoma IV}

En las larvas que mudaron al cuarto estado se observó que las anténulas, maxilas I y II y maxilípedos I a III, así como el tercer par de pereiópodos no presentaron modificaciones observables a microscopía de luz. Este último sólo aumenta su longitud y los cromatóforos esféricos aún están presentes en el extremo distal; adelante de la inserción del exopodito existen 2 espinas; el isquiomero y carpo terminan en 3 espinas divergentes y el propodito posee numerosas espinas ordenadas a distancias regulares en todo su contorno.

Aún cuando las estructuras anteriores son iguales en todas las larvas de este estado, se observó que algunas de ellas presentaban estructuras diferentes lo cual permite distinguir 2 sub-estados: IV A y IV B. Los principales cambios se observaron en la lon- gitud del cuarto y quinto par de pereiópodos y en el número de setas del exopodito del tercero.

IV A: Este sub-estado presenta un rostro de longitud menor que el ancho del pedúnculo ocular. El largo del cuarto pereiópodo equivale al doble de la longitud del abdomen (Fig. 6a). El exopodito del tercer par de pereiópodos presenta 4 o 5 pares de setas en su extremo distal. El quinto par es rudimentario, con una longitud equivalente a $1 / 4$ del largo del abdomen (Fig. 6c).

IV B: Este sub-estado difiere del anterior en que la longitud del rostro es mayor que el ancho del pedúnculo ocular y en el cuarto pereiópodo, que es 3,5 a 4 veces más largo que el abdomen (Fig. 6b) y presenta 5 pares de setas en su exopodito. El quinto par de pereiópodos es rudimentario y su longitud equivale entre $1 / 4$ a $1 / 2$ del largo del abdomen.

En ambos sub-estados el cuarto pereiópodo presenta un exopodito rudimentario que termina en 2 espinas divergentes; detrás de éstas, en el borde ventral, se insertan otras dos espinas divergentes similares a las anteriores; la coxa presenta una espina coxal rudimentaria en posición ventral. 


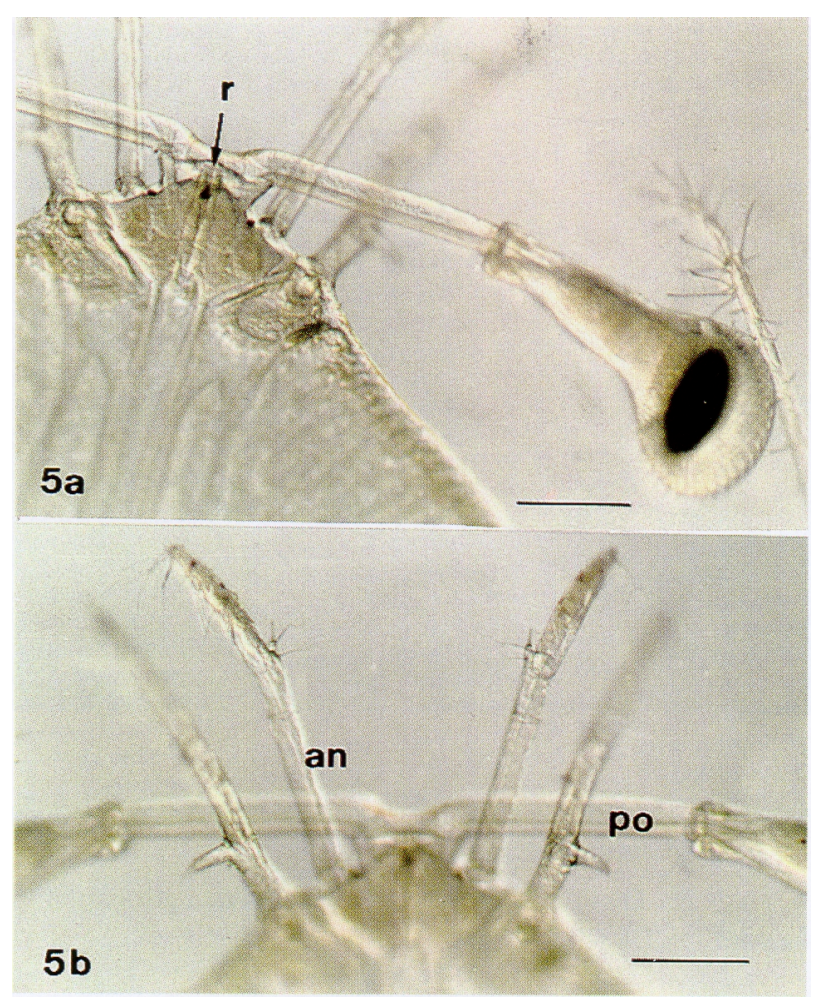

Figure 5. Estado III. a) y b) Extremo anterior de la larva; se observa antena (an) y anténula con una rama ventral corta, pedúnculos oculares (po) y rostro (r). Barras=0,3 mm.

Figure 3. Stage III. a) and b) Larvae in anterior view; are observed antenna (an) and antennule with a short ventral branch, ocular peduncles (po) and face (r). Bars $=0.3 \mathrm{~mm}$.

\section{Phyllosoma $V$}

El rostro se prolonga levemente por delante de los pedúnculos oculares. Los dos primeros pares de pereiópodos presentan 10 pares de setas en sus exopoditos nadadores. El tercer par sólo presenta 6 pares de setas en su exopodito. El cuarto par de pereiópodos presenta 3 segmentos, espina coxal desarrollada y exopodito rudimentario (Fig. 7). El quinto par es rudimentario y tiene longitud de $2 / 3$ (aprox. $45 \mu \mathrm{m}$ ) o de la misma longitud que el abdomen (Fig. 7); termina en una espina encorvada hacia el interior y en una seta que se inserta en el borde interno de la base de la espina. En el abdomen aparece una espina en el borde lateral externo de su región media. La antena continúa siendo bisegmentada y bífida al igual que el estado anterior; la anténula segmentada. El tamaño de la larva en este estado es notoriamente mayor que la larva del estado anterior (Fig. 8).

\section{Duración de los estados}

La duración de cada uno de los estados del desarrollo larval se señala en Tabla 2. No se observó una variación considerable ni en la duración de cada uno de los estados ni en la frecuencia de las mudas. El estado de phyllosoma I tuvo una duración de 12 a 15 días. La mayor frecuencia de mudas del estado I al II (56\%) ocurrió el día 12. La muda de los estados II se produjo entre los días 21 y 27 posteriores a la eclosión. La muda desde el estado III al IV se produjo entre los días 26 y 34 post-eclosión, observándose que la mayoría de los estados III cambiaron al estado IV-A y desde el estado IV al V entre los días 35 y 46. 

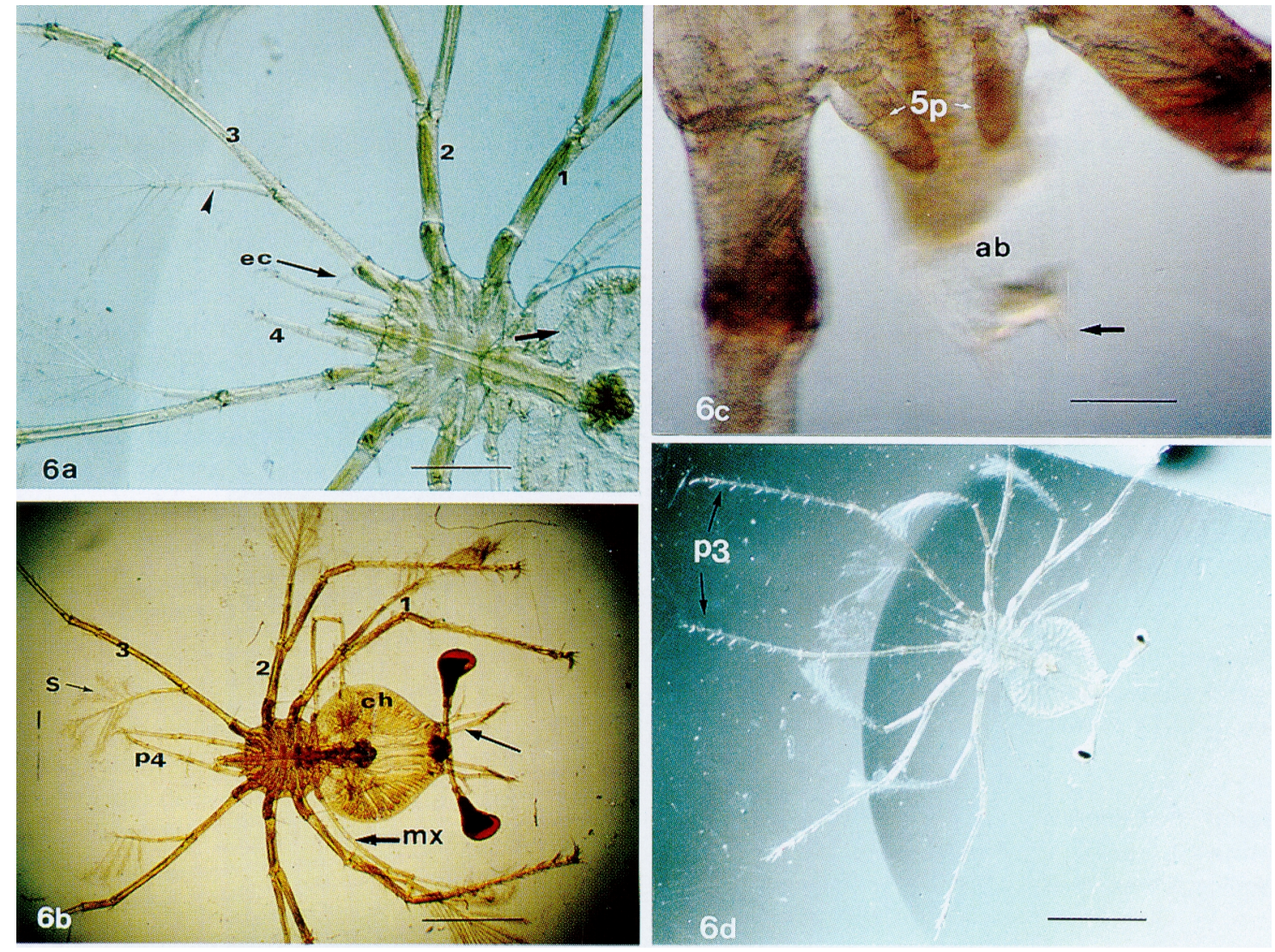

Figura 6. Estado IV. a) Sub-estado IV A, primero (1), segundo (2), tercero (3), y cuarto (4) pereiópodos; exopodito del tercer pereiópodo (punta de flecha) y espina coxal (ec). Barra=1 mm. b) Sub-estado IV B: se observa antena (flecha), ciegos hepáticos (ch), tercer maxilípedo (mx), primero (1), segundo (2), tercero (3) y cuarto (p4) pereiópopodos y setas (s) del exopodito del tercer pereiópodo. Barra=2 mm. c) Sub-estado IV A: vista ventral del abdomen (ab) que termina en un par de espinas romas (flecha) y quinto par de pereiópodos rudimentarios (5p). Barra =0,2 mm. d) Visión general del estado IV A; tercer par de pereiópodos (p3). Barra=2,7 mm.

Figure 6. Stage IV. a) Sub-stage IV A: first (1), second (2), third (3), and fourth (4) pereipodos; exopodite of the third pereiopod (arrow's end) and coxal spine (ec). Bar=1 mm. b) Sub-stage IV B: are observed antenna (arrow), hepatic cecae (ch), the third maxilliped (mx), first (1), second (2), third (3) and fourth (p4) pereiopods and setaes (s) of the exopodite in the third pereiopod. Bar=2 mm. c) Sub-stage IV A: ventral view of the abdomen (ab) that ends in a flat-nosed pair of spines (arrow) and fifth pair of rudimentary pereiopods (5p). Bar=0.2 mm; d) general view of stage IVA; third pair of pereiopods (p3). Bar=2.7 mm.

El período de intermuda más largo fue de 27 días a temperaturas de $18-23^{\circ} \mathrm{C}$.

La mayor mortalidad (83\%) se produjo entre el día 7 y 12 post-eclosión a temperaturas entre 20 y $23^{\circ} \mathrm{C}$.

\section{DISCUSION}

Báez (1973) refiriéndose a los estados de phyllosoma VII, XI y XIII de Jasus frontalis determinó que la longitud de sus cefalones varían entre los 9 y $27 \mathrm{~mm}$. En todas ellas el largo era menor que su ancho, lo cual sugiere que el cefalón varía sus proporciones longitud-ancho a medida que conti- 


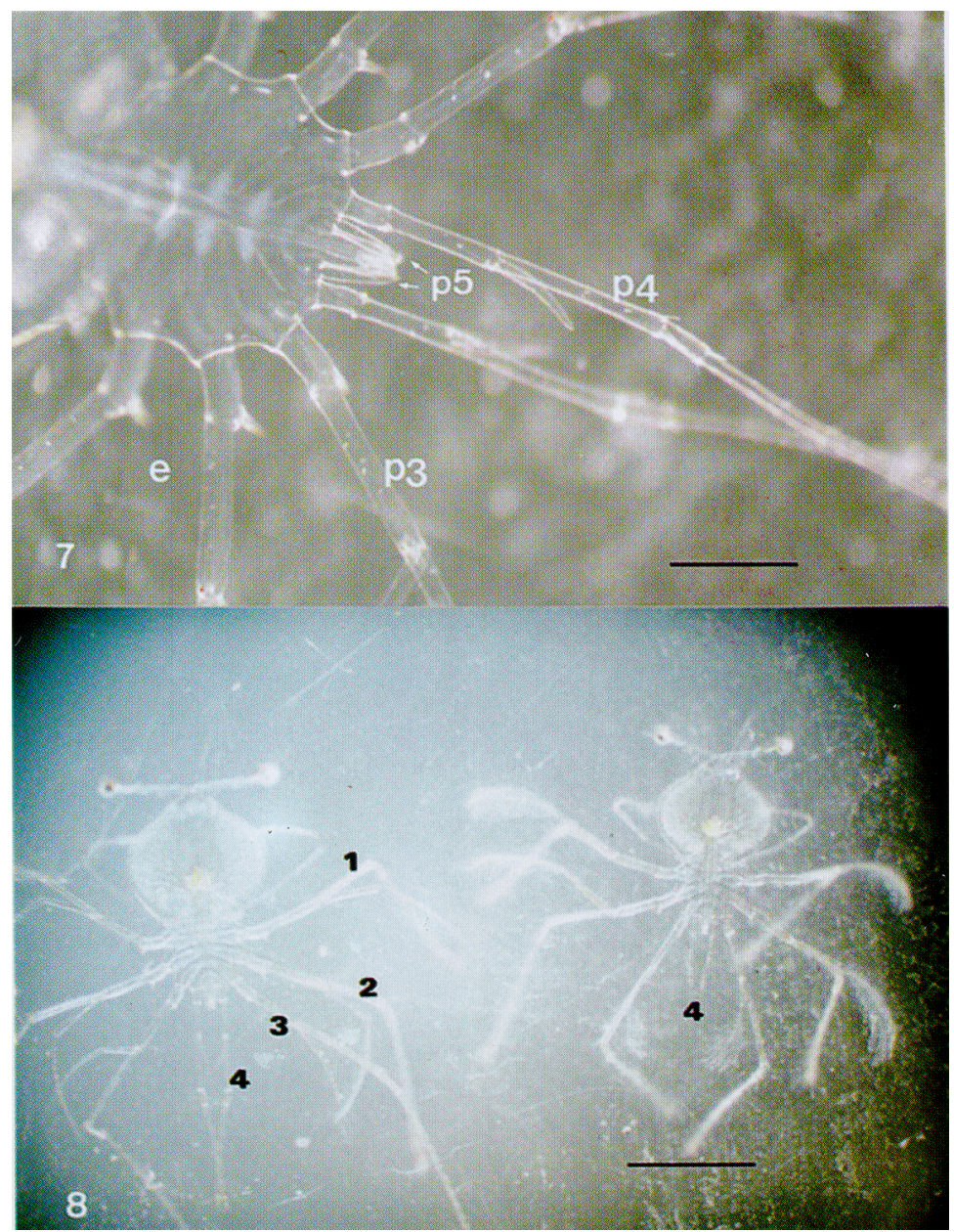

Figura 7. Estado V. Tercero (p3), cuarto (p4) y quinto (p5) par de pereiópodos. Barra=1 mm.

Figure 7. Stage V. Third (p3), fourth (p4) and fifth (p5) pair of pereiopods. Bar=1 mm.

Figura 8. Visión general de los estados V (izquierda) y IV (derecha); se observan los pereiópodos 1, 2, 3 y 4; nótese la diferencia de tamaño de ambas larvas y la disposición de los pedúnculos oculares. Barra=3 $\mathbf{~ m m}$.

Figure 8. General view of Stage V (left) and IV (right); pereiopods 1, 2, 3 and 4 are observed; note the size difference of both larvaes and positioning of the ocular peduncles. $B a r=3 \mathrm{~mm}$.

núa el desarrollo. En el estado I el ancho es mayor que el largo, en cambio, en el estado $\mathrm{V}$ ambas longitudes son semejantes. En los siguientes estados se hace más pronunciada la longitud ántero-posterior (Báez, 1973).

A pesar que el aspecto general de las phyllosomas de Panulirus interruptus (Johnson 1956) y J. frontalis son similares, el tamaño de la primera phyllosoma de $P$. interruptus es sólo de 1,44 $\mathrm{mm}$. Alcanza el tamaño del primer estado de $J$. frontalis $(2,2 \mathrm{~mm})$ sólo cuando aquellas llegan al estado IIIb (Dexter, 1972). Sin embargo, se ha establecido que el tamaño de los estados larvarios pro- 
venientes de cultivos presentan tamaños menores que aquellos capturados en el medio natural (Robertson 1968).

En Panulirus interruptus el estado II puede obtenerse dentro de los 7 días posteriores a la eclosión (Johnson 1956). En cambio, en el presente estudio se observó que en J. frontalis, el estado II ocurre dentro de los 12 a 15 días.

Si se considera que el promedio entre cada estado fue de 10 días a una temperatura promedio de $20^{\circ} \mathrm{C}$ y que el período de tiempo máximo en intermuda fue 27 días, es posible calcular el tiempo total que dura el desarrollo larval. Según Báez (1973), el número total de estados de desarrollo es 14, de modo que el desarrollo larval de Jasus frontalis, entre el estado de naupliosoma y puerulus, podría tener una duración entre 112 y 160 días y un tiempo máximo de 378 días a una temperatura de $20^{\circ} \mathrm{C}$.

Otro aspecto importante a considerar en un eventual cultivo de larvas es la alimentación, debido a que la mayor mortalidad se produjo en períodos variables antes de la primera muda. De acuerdo a Anger y Darwis (1981) y Criales y Anger (1968), la larva antes de mudar debe contar con una reserva de energía suficiente para permitir dicho proceso. También es importante determinar la relación que existe entre el número de sub-estados y la temperatura del cultivo y la interrelación de estas dos variables con la alimentación, ya que se estableció en Paleomonetes vulgaris, que la baja concentración de alimento tiene un efecto inhibitorio sobre el crecimiento pero no afecta el proceso de muda excepto cuando la temperatura es alterada (Knowlton, 1974).

En el presente estudio se determinó que el estado IV presentó dos sub-estados diferentes en cuanto al número de setas y longitud del cuarto par de pereiópodos. Esta variabilidad en los estados también ha sido establecido para otros grupos de Decapodos Caridea como Penaeus dourarum donde se observó entre 2 y 5 sub-estados (Edwald, 1965), sin embargo, debe ser investigado con mayor detalle.

\section{AGRADECIMIENTOS}

Agradezco a don Luis López, presidente del Sindicato de Pescadores de Juan Fernández y a Don Adrián Covarrubias, secretario de este Sindicato por la ayuda prestada en la realización de este estudio. Especialmente a Don Alvaro Elorza, a Don Albis González y Denis González por su valiosa ayuda en la captura de hembras con huevos y a todos aquellos amigos isleños que colaboraron en la obtención de ejemplares para este estudio.

\section{REFERENCIAS}

Báez, P. 1973. Larvas phyllosoma del Pacífico Sur Oriental (Crustacea, Macrura, Scyllaridea). Revista de Biología Marina, 15: 115-130.

Criales, M. y K. Anger. 1968. Experimental studies on the larval development of the shrimp Crangon crangon and C. allmanni. Helgolander Meeresunters, 40: 241-265.

Dexter, D. 1972. Molting and growth in laboratory reared phyllosomes of the California spiny lobster, Panulirus interruptus. California Fishery and Games 58:107-115.

Dupré, E. 1988a. Desarrollo embrionario de la langosta de Juan Fernández Jasus frontalis (Decápoda, Macrura, Palinuridae). Investigaciones Marinas 16: 49-62.

Dupré, E. 1988b. Cultivo de la langosta de Juan Fernández. I. Fecundación y crecimiento post larval. Informe final Proy. FONDECYT 1370/86.

Dupré, E. 1996. Primera phyllosoma de la langosta de Juan Fernández, Jasus frontalis. Rev. Chilena Hist. Nat., 60 (En prensa).

Edwald, J. 1965. The laboratory rearing of the pink shrimp, Penaeus dourarum Burkenroad. Bull. Mar. Sci., 15: 436-449.

Johnson, M. 1956. The larval development of the California spiny lobster, Panulirus interruptus (Randall), with notes on Panulirus gracillis. Proceedings of California Academy of Science, Serie 4, 26: 1-19.

Johnson, M. 1971. The phyllosoma of Scyllarus delfini (Bouvier) (Decapoda, Palinuridae). Crustaceana 21: 161-164.

Kittaka, J y E. Ikegami. 1988. Culture of the Palinurid Palinurus elephas from egg stage to puerulus. Nippon Suisan Gakkaishi 54 (7): 1149-1154. 
Kittaka, J. y K. Kimura. 1989. Culture of the Japanese spiny lobster Panulirus japonicus from egg to juvenile stage. Nippon Suisan Gakkaishi, 55: 963970.

Knowlton, R. 1974. Larval development processes and controlling factors in decapod Crustacea, with enphasis on Caridea. Thalassia Jugoslavica, 10: 138-158.

McWilliams, P. y B. Phillips. 1987. Distinguishing the phyllosome larvae of rock lobster species of the genus Jasus (Decapoda, Palinuridae) in the waters of Australia and New Zealand. Crustaceana 52: 1-24.

Recibido el 26 de junio de 1996.

Aceptado el 3 de diciembre de 1996.
Robertson, P. 1968. The complete larval development of the sand lobster Scyllarus americanus (Smith), (Decapoda, Syllaridae) in the laboratory with notes on the larvae from the plankton. Bulletin of Marine Science 18: 294-341.

Silberbauer, B. 1971. The biology of the South African rock lobster Jasus lalandii (H. Milne Edwards). I. The reproductive organs, mating and fertilization. Investigational Report Division Sea Fishery, South Africa 93: 1-46. 